\title{
EFFECT OF SEROTHERAPY DOSE ON IMMUNORECONSTITUTION FOLLOWING UMBILICAL CORD STEM CELL TRANSPLANT FOR PRIMARY IMMUNODEFICIENCY
}

P. Evans ${ }^{1}$, J.P. Lane ${ }^{1}$, M.A. Slatter ${ }^{1,2}$, D. Barge ${ }^{3}$, A. Jackson ${ }^{4}$, S. Hambleton ${ }^{1,2}$, T.J. Flood ${ }^{2}$, M. Abinun ${ }^{1,2}$, A.J. Cant ${ }^{1,2}$, A.R. Gennery ${ }^{1,2}$

${ }^{1}$ Institute of Cellular Medicine, University of Newcastle, ${ }^{2}$ Paediatric Immunology, Great North Children's Hospital, ${ }^{3}$ Immunology, Newcastle upon Tyne Hospital NHS Foundation Trust, ${ }^{4}$ Molecular Genetics, Newcastle upon Tyne Hospitals NHS Foundation Trust, Newcastle upon Tyne, UK

Background: Severe Primary Immunodeficiencies (PID) have high morbidity and mortality. Umbilical cord blood stem cell transplantation (UCSCT) is curative. Conditioning regimens aid engraftment and long-term thymopoiesis. Modification of cyto-reductive chemotherapy with or without anti-lymphocyte serotherapy may alter immunoreconstitution and outcome.

Aims: To examine speed of immunoreconstitution with respect to dose of serotherapy.

Methods: A retrospective review of time to neutrophil and platelet engraftment, $\geq 200 \mathrm{~T}$ cells $/ \mu 1$, thymopoiesis, and lymphocyte subsets at 2,4,6 and 12 months in 33 PID patients who received UCSCT between 1999-2010 in one centre.

Results: 12 patients received high dose $(>0.6 \mathrm{mg} / \mathrm{kg}), 10$ low dose, and 8 no serotherapy. Overall median time to neutrophil and platelet engraftment was 21 and 38 days respectively. There were significant differences in median lymphocyte counts between dosages of serotherapy at specific time periods post UCSCT, with a general trend of low dose resulting in higher counts than high dose or no serotherapy. Exceptions were CD3+ and CD4+ counts at 2 months when no serotherapy led to significantly higher counts. No serotherapy led to a significantly shorter time to $\geq 200 \mathrm{CD} 3$ cells $/ \mu 1$ (17.5 days, range $10-88, \mathrm{p}=0.001)$ but not to thymopoiesis. 6 evaluable patients achieved viral clearance by a median of 103 days post-UCSCT. Survival was $81 \%$.

Conclusions: This study shows that no serotherapy leads to higher lymphocyte counts by 2 months post UCSCT, but that higher lymphocyte counts are achieved with low dose serotherapy in the longer term. Further studies to determine mechanism are needed. 